\title{
Linear Precoder Design for Simultaneous Information and Energy Transfer Over Two-User MIMO Interference Channels
}

\author{
Ayça Özçelikkale, Member, IEEE, and Tolga M. Duman, Fellow, IEEE
}

\begin{abstract}
Communication strategies that utilize wireless media for simultaneous information and power transfer offer a promising perspective for efficient usage of energy resources. With this motivation, we focus on the design of optimal linear precoders for interference channels utilizing such strategies. We formulate the problem of minimizing the total minimum mean-square error while keeping the energy harvested at the energy receivers above given levels. Our framework leads to a non-convex problem formulation. For point-to-point multiple-input multiple-output channels, we provide a characterization of the optimal solutions under a constraint on the number of transmit antennas. For the general interference scenario, we propose two numerical approaches, one for the single antenna information receivers case, and the other for the general case. We also investigate a hybrid signalling scheme, where the transmitter sends a superposition of two signals: a deterministic signal optimized for energy transfer and an information carrying signal optimized for information and energy transfer. It is illustrated that if hybrid signalling is not incorporated into the transmission scheme, interference can be detrimental to the system performance when the number of antennas at the receivers is low.
\end{abstract}

Index Terms-Wireless power transfer, energy harvesting, simultaneous wireless information and power transfer (SWIPT), interference, linear precoding, MMSE.

\section{INTRODUCTION}

$\mathbf{E}$ FFICIENT usage of energy resources is a growing concern in today's communication systems. Solutions that consider energy harvesting $(\mathrm{EH})$ from radio-frequency signals instead of completely relying on batteries or the power from the grid offer a promising perspective. In these scenarios, wireless media is used for simultaneous information transmission and power transfer in contrast to performing each of these tasks separately. In this paper, we study transmission strategies to accomplish this as efficiently as possible. We focus on the

Manuscript received November 12, 2014; revised April 7, 2015; accepted May 26, 2015. Date of publication June 10, 2015; date of current version October 8, 2015. This publication was made possible by NPRP grant 4-12932-513 from Qatar National Research Fund (a member of Qatar Foundation). The statements made herein are solely the responsibility of the authors. The associate editor coordinating the review of this paper and approving it for publication was S. Jin.

A. Özçelikkale is with the Department of Signals and Systems, Chalmers University of Technology, Gothenburg SE-41296, Sweden (e-mail: ayca. ozcelikkale@chalmers.se).

T. M. Duman is with the Department of Electrical Engineering, Bilkent University, Ankara TR-06800, Turkey (e-mail: duman@ee.bilkent.edu.tr).

Color versions of one or more of the figures in this paper are available online at http://ieeexplore.ieee.org.

Digital Object Identifier 10.1109/TWC.2015.2443099 design of optimal linear precoders under the criterion of the minimum mean-square error (MMSE).

Much of the existing research on simultaneous wireless information and power transfer (SWIPT) is conducted with rate as the performance metric. Fundamental trade-offs between the rate and the energy for a single-input single-output point-topoint (P2P) additive white Gaussian noise (AWGN) channel is studied [1]. This framework is extended to AWGN channels with frequency selective fading [2]. Optimal transmission strategies are investigated for broadcast channels [3], [4], relay channels [5], [6] and interference channels [7]-[10]. Optimal power control problems are considered under rate considerations for various single-user and multi-user scenarios [11]. Practical code design solutions are investigated in [12].

Here we adopt an alternative approach and focus on linear precoding at the transmitters and linear filtering at the receivers. Proper design of precoders and filters have been shown to provide significant performance improvements in many different communication scenarios, see for instance [13]-[17] for a limited sample. It is noted that mean-square error filters provide a practical, but still reasonably accurate alternative for estimation of coded data symbols in contrast to maximum likelihood decoding [14]. Investigated scenarios cover a wide range of models, including point-to point channels [13], multiple-access channels [14] relay channels [15] and applications to robust designs [16], [17].

In linear precoder design, mean-square error or signal-tonoise ratio (SNR) based metrics are utilized as the typical performance criteria. Despite the above vast usage of these metrics for various communication scenarios, relatively small number of works that consider such metrics have appeared in the framework of energy harvesting. Most of these works focus on the scenarios where energy is harvested from possibly unreliable resources, but not necessarily from man-made wireless signals [18]-[20]. Contrary to these approaches focusing on the unreliable nature of the energy supply, here we consider another energy harvesting problem and focus on simultaneous transfer of energy and information. There have been a number of works focusing on SNR-based constraints for SWIPT systems, such as single-output multicast channel [21], interference channel [22], [23], and downlink scenario [24]. Unfortunately, these works typically focus single antenna receivers which limits the applicability of the results.

In this paper, we focus on multiple-input multiple-output (MIMO) interference channels. As an expository work, we first 


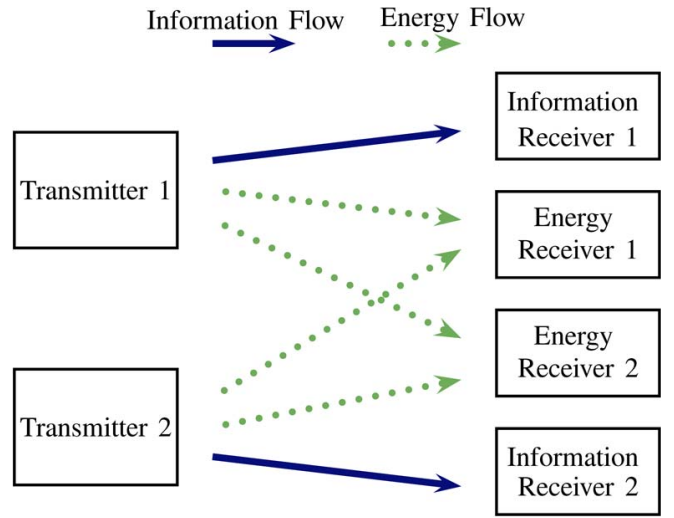

Fig. 1. Simultaneous information and energy transfer in two-user interference channel.

study the point-to-point channel with one information receiver (IR) and one energy receiver (ER). For this set-up, we formulate the problem of finding the optimal linear precoding strategy in order to minimize the MMSE at the information receiver while keeping the energy harvested at the energy receiver above a certain level. This formulation leads to a non-convex problem formulation. Nevertheless, under a constraint on the number of antennas at the transmitter, we provide a characterization of the optimal strategies that reveals the relationship between the channel matrices and the optimal transmission strategies. We also discuss the relationship between the rate maximization problem and the MMSE minimization problem. For the interference channel, we investigate the scenario illustrated in Fig. 1, where the transmitters aim to convey information as reliably as possible to their corresponding IRs while keeping the transferred power to the ERs above given levels. Here the transmitters have two possibly conflicting goals. One of these is keeping the interference at the non-designated IRs as low as possible to be able to transmit information reliably to the designated IRs. The other goal is to send as much power as possible to the ERs (which may be co-located with the IRs) in order to satisfy the EH constraints. We consider weighted sum MMSE as the performance criterion which leads to a non-convex optimization problem formulation. We propose two approaches for joint precoder design, where one of these approaches is developed solely for the single antenna IR case.

We also investigate a power splitting scheme at the transmitter, where the transmitted signal is the superposition of two signals where one of them is chosen to be deterministic and its sole purpose is to transfer power. We show that this scheme allows us to obtain smaller error values especially when the number of receive antennas is low. Contrary to the power splitting strategies for the receivers proposed in [3], here our aim is not to offer a feasible solution for the problem of practical $\mathrm{EH}$ receiver design problem. Instead, we illustrate that it is not always optimal to use a sole Gaussian signalling approach at the transmitter, see also [9] for similar discussions in the framework of rate maximization. This observation is important for understanding the fundamental limits of simultaneous information and power transfer. It shows that, even under the assumption of availability of ideal receivers that can simultaneously decode information and power, the signalling framework should be restructured to go beyond what Gaussian signalling offers.

We compare the performance of our designs with those of a time-division multiple-access (TDMA) approach and a time-division mode switching (TDMS) approach. These comparisons are motivated by the fact that schemes that depend on such mode separations have been considered as practical benchmarks in the interference channel in the context of rate maximization [7]-[10]. We illustrate that our proposed designs outperform the TDMA and TDMS approaches in low to moderate interference scenarios. Nevertheless, we note that in the case of co-located IR and ERs, our designs are based on an ideal receiver structure, i.e., the receiver can harvest energy and decode information simultaneously. It is not clear whether this receiver structure can be realized [3], hence in these scenarios our transmission schemes should be interpreted as designs for the baseline performance.

The rest of the paper is organized as follows. The system model for the interference channel and the joint linear precoder design problem are presented in Section II and Section III, respectively. In Section IV, we present the special case that focuses on the point-to-point channel. In Section V and in Section VI, our joint linear precoder design approach for the interference channel is presented for the single antenna IR case and the general case, respectively. We discuss the hybrid signalling approach in Section VII. The performance of our designs are illustrated in Section VIII. We conclude the paper in Section IX.

The following notation is used throughout the paper. Uppercase and lowercase letters denote matrices, and column/row vectors respectively. The complex conjugate transpose of a matrix $A$ is denoted by $A^{\dagger}$. The operators $E[$.$] , and \operatorname{tr}[$.$] de-$ note the expectation, and trace operators respectively. $\operatorname{diag}(a)$ denotes the diagonal matrix formed with $a$ as the diagonal elements. $I$ denotes the identity matrix with the suitable dimensions. Positive semi-definite ordering is denoted by $\succeq$, where $A \succeq 0$ denotes a Hermitian positive semi-definite matrix. An optimal value of an optimization variable $A$ is denoted by $A^{*}$.

\section{System Model}

\section{A. Interference Channel}

The multi-antenna transmitters transfer information to information receivers as well as power to energy harvesting receivers according to the following model

$$
\begin{aligned}
y_{i}^{I} & =H_{i 1}^{I} x_{1}+H_{i 2}^{I} x_{2}+w_{i}^{I}, \\
y_{i}^{E} & =H_{i 1}^{E} x_{1}+H_{i 2}^{E} x_{2}+w_{i}^{E},
\end{aligned}
$$

where $i=1,2$. Here $H_{i k}^{I} \in \mathbb{C}^{n_{r} \times n_{t}}$ and $H_{i k}^{E} \in \mathbb{C}^{n_{e} \times n_{t}}$ represent the channel gains from the transmitter $k$ to information receiver $i\left(\mathrm{IR}_{i}\right)$ and energy receiver $i\left(\mathrm{ER}_{i}\right)$, respectively $i, k=1,2$. This system is illustrated in Fig. 1. Zero-mean complex proper Gaussian $w_{i}^{I} \in \mathbb{C}^{n_{r} \times 1} \sim \mathcal{C N}\left(0, K_{w_{i}^{I}}\right), K_{w_{i}^{I}}=$ $E\left[w_{i}^{I}\left(w_{i}^{I}\right)^{\dagger}\right]=\sigma_{w, I, i}^{2} I$ and $w_{i}^{E} \in \mathbb{C}^{n_{e} \times 1} \sim \mathcal{C N}\left(0,, K_{w_{i}^{E}}\right), K_{w_{i}^{E}}=$ $E\left[w_{i}^{E}\left(w_{i}^{E}\right)^{\dagger}\right]=\sigma_{w, E, i}^{2} I$ denote the noise at IR's and ER's channels, respectively. 
The channel input $x_{i}$ is formed as $x_{i}=A_{i} s_{i}$, where the zero mean complex proper Gaussian $s_{i} \in \mathbb{C}^{n \times 1}, s_{i} \sim \mathcal{C N}\left(0, K_{s_{i}}\right)$ $K_{s_{i}}=I$, denotes the data, $A_{i} \in \mathbb{C}^{n_{t} \times n}$ denotes the precoding matrix at the $i^{\text {th }}$ transmitter. All signals, $w_{i}^{I}, w_{i}^{E}$, and $s_{i}$, are assumed to be statistically independent. All channel gains are fixed throughout the transmission.

\section{B. MMSE Estimation}

The designated information receiver for transmitter $i$ is the receiver $i$. Hence upon receiving $y_{i}^{I}, \mathrm{IR}_{i}$ forms an estimate of $s_{i}$. We assume that IRs employ MMSE estimation. Hence the estimate of $s_{i}$ at IR $\mathrm{IR}_{i}$ can be expressed as follows [25, Ch. 2]:

$$
E\left[s_{i} \mid y_{i}^{I}\right]=K_{s_{i} y_{i}^{I}} K_{y_{i}^{I}}^{-1} y_{i}^{I},
$$

where $K_{s_{i} y_{i}^{I}}=E\left[s_{i} y_{i}^{I^{\dagger}}\right]=K_{s_{i}} A_{i}^{\dagger} H_{i i}^{I^{\dagger}}$, and $K_{y_{i}^{I}}=E\left[y_{i}^{I} y_{i}^{I^{\dagger}}\right]=$ $H_{i i}^{I} A_{i} A_{i}^{\dagger} H_{i i}^{I^{\dagger}}+H_{i j}^{I} A_{j} A_{j}^{\dagger} H_{i j}^{I^{\dagger}}+\sigma_{w, I, i}^{2}$. We note that since $\sigma_{w, I, i}^{2} I \succ 0$, we have $K_{y_{i}} \succ 0$, and hence $K_{y_{i}}^{-1}$ exists. The MMSE at $\mathrm{IR}_{i}$ can be expressed as follows

$$
\begin{aligned}
\varepsilon_{i}\left(A_{1}, A_{2}\right) & =E\left[\left\|s_{i}-E\left[s_{i} \mid y_{i}^{I}\right]\right\|^{2}\right] \\
& =\operatorname{tr}\left[K_{S_{i}}-K_{s_{i} y_{i}^{I}} K_{y_{i}^{I}}^{-1} K_{s_{i} y_{i}^{\prime}}^{\dagger}\right] \\
& =n-\operatorname{tr}\left[A_{i}^{\dagger} H_{i i}^{I^{\dagger}}\left(T_{i}+\sigma_{w, I, i}^{2}\right)^{-1} H_{i i}^{I} A_{i}\right],
\end{aligned}
$$

where $T_{i}=H_{i i}^{I} A_{i} A_{i}^{\dagger} H_{i i}^{I^{\dagger}}+H_{i j}^{I} A_{j} A_{j}^{\dagger} H_{i j}^{I^{\dagger}}, i, j=1,2, i \neq j$.

\section{Energy Harvesting}

The energy harvested at the $\mathrm{ER}_{i}$ can be expressed as [3]

$\mathcal{J}_{i}\left(A_{1}, A_{2}\right)=\beta\left(\operatorname{tr}\left[H_{i 1}^{E} A_{1} A_{1}^{\dagger} H_{i 1}^{E^{\dagger}}\right]+\operatorname{tr}\left[H_{i 2}^{E} A_{2} A_{2}^{\dagger} H_{i 2}^{E^{\dagger}}\right]\right)$,

where $0 \leq \beta \leq 1$ accounts for the possible loss in the energy conversion process. Without loss of generality, we assume that this loss is accounted for while setting desired energy levels and hence we use $\beta=1$ in our formulations.

\section{LINEAR PRECODER DESIGN}

We consider the following joint linear precoder design problem which seeks the optimal linear precoders in order to minimize the weighted MMSE at the information receivers while satisfying the energy requirements at the energy receivers:

$$
\begin{array}{ll}
\text { (P1) } \min _{A_{1}, A_{2}} & \alpha_{1} \varepsilon_{1}\left(A_{1}, A_{2}\right)+\alpha_{2} \varepsilon_{2}\left(A_{1}, A_{2}\right) \\
\text { s.t. } & \mathcal{J}_{1}\left(A_{1}, A_{2}\right) \geq \gamma_{1}, \quad \mathcal{J}_{2}\left(A_{1}, A_{2}\right) \geq \gamma_{2}, \\
& \operatorname{tr}\left[A_{1} A_{1}^{\dagger}\right] \leq P_{1}, \quad \operatorname{tr}\left[A_{2} A_{2}^{\dagger}\right] \leq P_{2} .
\end{array}
$$

Here (6c) represents the power constraints at the transmitters. The scalars $\alpha_{1}$ and $\alpha_{2}$ represent the error weights for different users. These weights can be used to prioritize one of the IRs in the system, for instance a large $\alpha_{1} / \alpha_{2}$ ratio will give a large penalty to the estimation error at $\mathrm{IR}_{1}$, hence will result in strategies that favor $\mathrm{IR}_{1}$. Alternative formulations for prioritizing different users can be also adopted, see for instance [26] where energy efficiency in an interference channel scenario is studied.

We now discuss the convexity properties of the formulation in Problem $P 1$. Using the property $\operatorname{tr}[A B]=\operatorname{tr}[B A]$, the energy harvesting constraints can be equivalently written as

$$
\mathcal{J}_{i}\left(A_{1}, A_{2}\right)=\operatorname{tr}\left[A_{1}^{\dagger} H_{i 1}^{E^{\dagger}} H_{i 1}^{E} A_{1}\right]+\operatorname{tr}\left[A_{2}^{\dagger} H_{i 2}^{E^{\dagger}} H_{i 2}^{E} A_{2}\right],
$$

which is a quadratic function in $\left(A_{1}, A_{2}\right)$. Moreover, since $H_{i j}^{E^{\dagger}} H_{i j}^{E}, i, j=1,2$, are positive semi-definite, $\mathcal{J}_{i}\left(A_{1}, A_{2}\right)$ is a convex quadratic function. Hence the $\mathrm{EH}$ constraints form nonconvex constraints since they bound convex functions from below. It is worth mentioning that the objective function is also not a convex function of $\left(A_{1}, A_{2}\right)$, which is true even for the scalar case.

We now illustrate that even when the traditional semi-definite rank relaxations are introduced, the resulting optimization problem is still non-convex. We introduce the following new variables of optimization: $K_{i}=A_{i} A_{i}^{\dagger}, i=1,2$. Hence the energy harvested at the ERs can be expressed as linear functions of $\left(K_{1}, K_{2}\right)$

$$
\mathcal{J}_{i}^{K}\left(K_{1}, K_{2}\right)=\operatorname{tr}\left[H_{i 1}^{E} K_{1} H_{i 1}^{E^{\dagger}}\right]+\operatorname{tr}\left[H_{i 2}^{E} K_{2} H_{i 2}^{E^{\dagger}}\right] .
$$

Furthermore, it is possible to write the objective function in terms of $\left(K_{1}, K_{2}\right)$, for instance by using the property $\operatorname{tr}[A B]=$ $\operatorname{tr}[B A]$ on $(4 \mathrm{c})$ as follows

$$
\varepsilon_{i}^{K}\left(K_{1}, K_{2}\right)=n-\operatorname{tr}\left[H_{i i}^{I} K_{i}^{\dagger} H_{i i}^{I^{\dagger}}\left(T_{i}^{K}+\sigma_{w, I, i}^{2} I\right)^{-1}\right],
$$

where $T_{i}^{K}=H_{i i}^{I} K_{i} H_{i i}^{I^{\dagger}}+H_{i j}^{I} K_{j} H_{i j}^{I^{\dagger}}$ and $i, j=1,2, i \neq j$. We note that now $K_{i}$ should have a decomposition such that $K_{i}=A_{i} A_{i}^{\dagger}, A_{i} \in \mathbb{C}^{n_{t} \times n}$, i.e., we have rank constraints on the variables $K_{i}, \operatorname{rank}\left(K_{i}\right) \leq n$. By lifting these constraints, one may form the following relaxed optimization problem

$$
\begin{array}{cl}
(\bar{P} 1) \min _{K_{1} \succeq 0, K_{2} \succeq 0} & \alpha_{1} \varepsilon_{1}^{K}\left(K_{1}, K_{2}\right)+\alpha_{2} \varepsilon_{2}^{K}\left(K_{1}, K_{2}\right) \\
\text { s.t. } \quad & \mathcal{J}_{1}^{K}\left(K_{1}, K_{2}\right) \geq \gamma_{1}, \quad \mathcal{J}_{2}^{K}\left(K_{1}, K_{2}\right) \geq \gamma_{2}, \\
& \operatorname{tr}\left[K_{1}\right] \leq P_{1}, \quad \operatorname{tr}\left[K_{2}\right] \leq P_{2} .
\end{array}
$$

Hence Problem $\bar{P} 1$ forms a semi-definite programming (SDP) relaxation of Problem $P 1$. Further information about such rank relaxations can be found in [27]-[29]. Although now all the constraints form convex constraints, this formulation is still non-convex, since the objective function in (10a) is a not a convex function of $\left(K_{1}, K_{2}\right)$ for the general interference channel scenario. An exception is the case of point-to-point channel, where there is only one user (but the channel may still be a MIMO channel). For this case, by using convexity, we provide a semi-explicit analytic characterization of the solutions in Section IV for the case $n_{t} \leq n$. Starting with Section V, we focus on the interference channel. 


\section{Point-To-Point ChanNel}

In this section, we consider the scenario where there is only transmitter with one designated IR and one designated ER. We assume that $x_{2}=0$ without loss of generality. Hence transmitter 1 sends data to $I_{1}$ as well as power to $E_{1}$

$$
\begin{aligned}
& y_{1}^{I}=H_{11}^{I} x_{1}+w_{1}^{I}, \\
& y_{1}^{E}=H_{11}^{E} x_{1}+w_{1}^{E},
\end{aligned}
$$

where $H_{11}^{I}, H_{11}^{E}, w_{1}^{I}, w_{1}^{E}$, and $x_{1}$ are as defined in Section II-A. We focus on the case $n_{t} \leq n$. The MMSE at $\mathrm{IR}_{1}$ can be expressed as

$$
\begin{aligned}
\varepsilon_{1}\left(A_{1}, 0\right) & =\operatorname{tr}\left[I-A_{1}^{\dagger} H_{11}^{I^{\dagger}}\left(H_{11}^{I} A_{1} A_{1}^{\dagger} H_{11}^{I^{\dagger}}+\sigma_{w, I, 1}^{2} I\right)^{-1} H_{11}^{I} A_{1}\right] \\
& =\operatorname{tr}\left[\left(I+\frac{1}{\sigma_{w, I, 1}^{2}} A_{1}^{\dagger} H_{11}^{I^{\dagger}} H_{11}^{I} A_{1}\right)^{-1}\right],
\end{aligned}
$$

where (13) follows from Sherman-Morrison-Woodbury identity [30]. The energy harvested at the $\mathrm{ER}_{1}$ can be expressed as

$$
\mathcal{J}_{1}\left(A_{1}, 0\right)=\operatorname{tr}\left[H_{11}^{E} A_{1} A_{1}^{\dagger} H_{11}^{E^{\dagger}}\right] .
$$

We consider the problem of minimization of the MMSE at the $\mathrm{IR}_{1}$ while satisfying the $\mathrm{EH}$ constraint at the $\mathrm{ER}_{1}$

(P2) $\min _{A_{1}} \varepsilon\left(A_{1}, 0\right)$

$$
\text { s.t. } \quad \mathcal{J}_{1}\left(A_{1}, 0\right) \geq \gamma_{1}, \quad \operatorname{tr}\left[A_{1} A_{1}^{\dagger}\right] \leq P_{1} .
$$

This formulation is non-convex. Nevertheless, for $n_{t} \leq n$, it is possible to characterize the optimal solutions for (15) by introducing an equivalent formulation that is convex and whose solutions in fact provide optimal solutions for (15).

Utilizing [31, Lem. 2], we express the problem of minimizing (13) equivalently as the problem of minimizing $\operatorname{tr}\left[\left(I+\left(1 / \sigma_{w, I, 1}^{2}\right) H_{11}^{I} A_{1} A_{1}^{\dagger} H_{11}^{I^{\dagger}}\right)^{-1}\right]$. Hence we arrive at the following optimization problem by introducing $K_{1}=A_{1} A_{1}^{\dagger}$

$$
\begin{array}{ll}
\text { (P3) } \min _{K_{1} \succeq 0} \operatorname{tr}\left[\left(I+\frac{1}{\sigma_{w, I, 1}^{2}} H_{11}^{I} K_{1} H_{11}^{I^{\dagger}}\right)^{-1}\right] \\
\text { s.t. } \quad \operatorname{tr}\left[H_{11}^{E} K_{1} H_{11}^{E^{\dagger}}\right] \geq \gamma_{1}, \quad \operatorname{tr}\left[K_{1}\right] \leq P_{1} .
\end{array}
$$

This is a convex optimization problem whose convexity can be established as follows: i) The objective function is a convex function of $K_{1}$ since $f(X)=\operatorname{tr}\left[X^{-1}\right]$ is convex over $X \succ 0$ [32]; ii) The inequality constraints are linear, hence convex. Since this is a convex SDP problem, an optimal solution can be found numerically by using off-the-shelf numerical optimization tools such as SeDuMi, SDPT3 and CVX [33]-[35]. Instead, here we utilize the solutions of Problem P3 to arrive at a characterization of the solutions of Problem P2.

Theorem 4.1: Let $n_{t} \leq n$. Assume that there exists a strictly feasible $A_{1}$ for Problem P2. Then an optimal solution has the following form:

$$
A_{1}^{*}=R^{-1 / 2} V_{\bar{H}} \Lambda^{1 / 2},
$$

where $\Lambda=\operatorname{diag}\left(\lambda_{i}\right)$ with $\lambda_{i}$ given by the following water-filling type solution

$$
\lambda_{i}=\left[\sqrt{\frac{1}{\lambda_{\bar{H}, i}}}-\frac{1}{\lambda_{\bar{H}, i}}\right]^{+},
$$

where $[x]^{+}=\max (0, x)$. Here $\quad R=\mu_{p} I-\mu_{e} H_{11}^{E_{1}^{\dagger}} H_{11}^{E}$, $\mu_{p}, \mu_{e} \geq 0$ and $\bar{H}=R^{-1 / 2} H_{11}^{I^{\dagger}} H_{11}^{I} R^{-1 / 2}=V_{\bar{H}} \Lambda_{\bar{H}} V_{\bar{H}}^{\dagger}$ is the singular value decomposition of $\bar{H}$, where $\Lambda_{\bar{H}}=\operatorname{diag}\left(\lambda_{\bar{H}, i}\right)$, $\lambda_{\bar{H}, 1} \geq \lambda_{\bar{H}, 2}, \ldots, \lambda_{\bar{H}, m}, m=\operatorname{rank}[\bar{H}]$.

The proof relies on the solution of the dual problem for Problem P3 and the fact that it is always possible to find an optimal $A_{i}$ from an optimal $K_{i}$ due to $n_{t} \leq n$. This line of arguments has been successfully adopted to reveal structures of the optimal solutions in a number of scenarios [3], [32], [36]. In particular, we refer the reader to [3] for the solution of rate maximization under EH constraints. Here we omit the proof for the MMSE case for the sake of brevity.

The result reveals that optimal solutions lie in the span of the right singular vectors of the modified channel matrix $H_{11}^{I} R^{-1 / 2}$. This result also illustrates the relationship between the rate maximization problem investigated in [3] and the MMSE minimization problem investigated here: the general structure of the solutions are similar where the eigenvectors of the optimal transmit covariance matrix $K_{1}$ lie in the span of the singular vectors of a matrix in the form $H_{11}^{I} R^{-1 / 2}, R=\mu_{p} I-\mu_{e} H_{11}^{E^{\dagger}} H_{11}^{E}$ where $\mu_{p}, \mu_{e}$ may take possibly different values for the MMSE minimization and rate maximization. Another related issue is the use of weighted MMSE criterion as an intermediate step in rate maximization problem, as explored in [37] without the $\mathrm{EH}$ constraints. Our result here can be utilized while formulating a similar relationship for SWIPT systems.

As discussed earlier, the linear precoder design problem with MMSE minimization, in general, has a rank constraint: $K_{1}$ should have a decomposition such that $K_{1}=A_{1} A_{1}^{\dagger}, A_{1} \in$ $\mathbb{C}^{n_{t} \times n}$. On the other hand, in the case of rate maximization, there is no such constraint, the transmit covariance matrix $K_{1}$ is the sole variable of interest [3, Problem P2]. Although a solution for an optimal $A_{1}$ satisfying rank constraints can be always found without putting any restrictions on the number of transmit antennas in the case of MMSE minimization without the EH constraints [13], [16], whether this is the case under EH constraints is not clear. We note that, even in the case with $n_{t} \leq n$, the designs optimized for these two metrics (rate and the MMSE) lead to different MMSE performance (with the exception of the case $n_{r}=1$, where these metrics lead to equivalent objective functions). This performance gap is illustrated in Section VIII. 


\section{INTERFERENCE SCENARIO: MISO INFORMATION CHANNEL}

We now consider the interference scenario with multiple-input single-output (MISO) information channel, i.e., Problem $P 1$ with $n_{r}=1$. As discussed in Section III, neither the general problem with multiple antenna IRs nor the single antenna scenario result in convex formulations. Nevertheless, here we propose a method to solve Problem $P 1$ for the MISO case using a sequence of convex problems.

In the following we will first consider the relaxed problem, Problem $\bar{P} 1$ in (10), and ignore the constraints $K_{i}=A_{i} A_{i}^{\dagger}, i=$ 1,2 . We will first focus on finding optimal $K_{i}$ 's, then we will discuss how to find optimal $A_{i}$ 's for Problem $P 1$ in Lemma 5.1. Under MISO information channel scenario, the MMSE at $\mathrm{IR}_{i}$ can be specialized to the following expression

$$
\varepsilon_{i}^{K}\left(K_{1}, K_{2}\right)=n-\frac{h_{i i}^{I} K_{i} h_{i i}^{I^{\dagger}}}{h_{i i}^{I} K_{i} h_{i i}^{I^{\dagger}}+h_{i j}^{I} K_{j} h_{i j}^{I^{\dagger}}+\sigma_{w, I, i}^{2}} .
$$

Here we have used lower case letters for the channel matrices to emphasize that they can now be represented as row vectors. Energy harvested at the ERs are given as in (8).

We now consider Problem $\bar{P} 1$. We recall that although the EH and power constraints form convex constraints, Problem $\bar{P} 1$ is not convex. As seen in (19), the individual terms $\varepsilon_{i}^{K}\left(K_{1}, K_{2}\right)$, $i=1,2$ in the objective function are linear fractional functions in $\left(K_{1}, K_{2}\right)$, hence they are not convex. In order to solve this non-convex optimization problem, we propose the following approach which utilizes the additional variable $\kappa$

$$
\begin{array}{cll}
\left(\bar{P} 1_{S O}\right) \min _{\kappa_{L} \leq \kappa \leq \kappa_{U}} & \min _{K_{1} \succeq 0, K_{2} \succeq 0} & \alpha_{1} \varepsilon_{1}^{K}\left(K_{1}, K_{2}\right)+\alpha_{2} \kappa \\
& \text { s.t. } & \varepsilon_{2}^{K}\left(K_{1}, K_{2}\right) \leq \kappa,
\end{array}
$$

subject to (10b) and (10c). Here $\kappa_{L}=n-1$ and $\kappa_{U}=n$, which are the lower and upper bounds on the MMSE for a singleoutput antenna system with signal power $\operatorname{tr}\left(K_{s_{2}}\right)=n$. We note that $\left(\bar{P} 1_{S O}\right)$ with the additional variable $\kappa$ is equivalent to the Problem $\bar{P} 1$; further discussions on this type of transformations can be found in [32, Ch. 4]. Here (20b) can be equivalently written as a linear inequality constraint

$$
-h_{22}^{I} K_{2} h_{22}^{I^{\dagger}}+\bar{\kappa}\left(h_{21}^{I} K_{1} h_{21}^{I^{\dagger}}+\sigma_{w, I, 2}^{2}\right) \leq 0
$$

where $\bar{\kappa} \doteq(n-\kappa) /(1-n+\kappa)$.

Let us now consider the inner minimization problem in Problem $\bar{P} 1_{S O}$. After straightforward algebraic manipulations, it can be expresssed as follows for a given $\kappa$

$$
\max _{K_{1}, K_{2}} \frac{h_{11}^{I} K_{1} h_{11}^{I^{\dagger}}}{h_{12}^{I} K_{2} h_{12}^{I^{\dagger}}+\sigma_{w, I, 1}^{2}}
$$

subject to (10b), (10c), and (21). The objective function is still in linear fractional form, hence the problem is not convex. To obtain a convex formulation, we utilize Charnes-Cooper transform [38] and define the following new variables:

$$
\begin{aligned}
t & =\left(h_{12}^{I} K_{2} h_{12}^{I^{\dagger}}+\sigma_{w, I, 1}^{2}\right)^{-1}, \\
\bar{K}_{1} & =t K_{1}, \quad \bar{K}_{2}=t K_{2} .
\end{aligned}
$$

Rewriting the optimization problem in (22) in terms of these variables, we arrive at the following formulation

$$
\begin{array}{ll}
\max _{K_{1} \succeq 0, K_{2} \geq 0, t \geq 0} & h_{11}^{I} \bar{K}_{1} h_{11}^{I^{\dagger}} \\
\text { s.t. } & h_{12}^{I} \bar{K}_{2} h_{12}^{I^{\dagger}}+t \sigma_{w, I, 1}^{2}=1, \\
& -h_{22}^{I} \bar{K}_{2} h_{22}^{I^{\dagger}}+\bar{\kappa}\left(h_{21}^{I} \bar{K}_{1} h_{21}^{I^{\dagger}}+t \sigma_{w, I, 2}^{2}\right) \leq 0, \\
& \mathcal{J}_{1}\left(\bar{K}_{1}, \bar{K}_{2}\right) \geq t \gamma_{1}, \quad \mathcal{J}_{2}\left(\bar{K}_{1}, \bar{K}_{2}\right) \geq t \gamma_{2}, \\
& \operatorname{tr}\left[\bar{K}_{1}\right] \leq t P_{1}, \quad \operatorname{tr}\left[\bar{K}_{2}\right] \leq t P_{2} .
\end{array}
$$

We observe that under mild conditions it is possible to construct optimal precoders $\left(A_{1}^{*}, A_{2}^{*}\right)$ from an optimal $\left(\bar{K}_{1}^{*}, \bar{K}_{2}^{*}\right)$ :

Lemma 5.1: Let $n \geq 2$. Assume that the optimization problem in (25) and its dual are solvable. Then an optimal solution in terms of $\left(A_{1}^{*}, A_{2}^{*}, t^{*}\right)$ can be always formed from an optimal solution $\left(\bar{K}_{1}^{*}, \bar{K}_{2}^{*}, t^{*}\right)$.

The proof is given in Appendix A. Now a solution to Problem $P 1$ under the MISO scenario can be found by solving Problem $\bar{P} 1_{S O}$. The solution to Problem $\bar{P} 1_{S O}$ will be found using a line search over $\kappa$ and the solution of inner optimization problem, i.e., (22) or equivalently (25). By Lemma 5.1, optimal precoders $\left(A_{1}^{*}, A_{2}^{*}\right)$ can be found from an optimal solution of (25). The optimization problem in (25) is convex in $\left(\bar{K}_{1}, \bar{K}_{2}, t\right)$, hence it can be solved by using available solvers, such as [33]-[35]. We note that the inner optimization problem formulates a scenario which may be of independent interest. Here the error performance for one of the users is optimized under a performance guarantee for the other user.

\section{INTERFERENCE SCENARIO: MIMO INFORMATION CHANNEL}

We now consider the general MIMO information channel scenario. We propose an alternating minimization technique for the solution of Problem $P 1$. We first consider the fixed receiver estimator case in Section VI-A. In Section VI-B, we utilize this scenario to provide linear precoder designs for the MMSE receiver case.

\section{A. Fixed Estimator at the Receiver}

Let $B_{i}$ be the estimator at $\mathrm{IR}_{i}$. Hence the mean-square error at $\mathrm{IR}_{i}$ can be expressed as follows:

$$
\begin{aligned}
\varepsilon_{i}^{F}= & E\left[\left\|s_{i}-B_{i} y_{i}^{I}\right\|^{2}\right], \\
= & \operatorname{tr}\left[K_{s_{i}}\right]-\operatorname{tr}\left[K_{s_{i} y_{i}^{I}} B_{i}^{\dagger}\right]-\operatorname{tr}\left[B_{i} K_{s_{i} y_{i}^{I}}^{\dagger}\right]+\operatorname{tr}\left[B_{i} K_{y_{i}^{I}} B_{i}^{\dagger}\right], \\
= & n-\operatorname{tr}\left[A_{i}^{\dagger} H_{i i}^{I^{\dagger}} B_{i}^{\dagger}\right]-\operatorname{tr}\left[B_{i} H_{i i}^{I} A_{i}\right]+\operatorname{tr}\left[B_{i} H_{i i}^{I} A_{i} A_{i}^{\dagger} H_{i i}^{I^{\dagger}} B_{i}^{\dagger}\right] \\
& +\operatorname{tr}\left[B_{i} H_{i j}^{I} A_{j} A_{j}^{\dagger} H_{i j}^{I^{\dagger}} B_{i}^{\dagger}\right]+\sigma_{w, I, i}^{2} \operatorname{tr}\left[B_{i} B_{i}^{\dagger}\right] .
\end{aligned}
$$

where $i, j=1,2, i \neq j$. Hence, for fixed receiver filters, the problem of finding the optimal linear precoders in order to 
minimize weighted sum of the estimation errors can be formulated as follows

$$
\min _{A_{1}, A_{2}} \quad \alpha_{1} \varepsilon_{1}^{F}+\alpha_{2} \varepsilon_{2}^{F}
$$

subject to (6b) and (6c). We note that the objective and the constraint functions are quadratic functions in $\left(A_{1}, A_{2}\right)$, hence this is a quadratically constrained quadratic programming (QCQP) problem. In general, QCQP problems are known to be NP hard even for the formulations in which objective function is convex and there is only one vector optimization variable [39] as opposed to the more involved case of two matrix variables here.

As discussed earlier, the EH constraints are not convex in $\left(A_{1}, A_{2}\right)$. To deal with these constraints, we introduce new variables $Z_{i}=A_{i} A_{i}^{\dagger}, i=1,2$. (Here we refrain from using the notation $K_{i}=A_{i} A_{i}^{\dagger}$ to avoid confusion with the previous formulations in Sections IV and V where it is possible to write the whole optimization problem in terms of $K_{i}$.) Ignoring the constant terms, we rewrite the part of the error that depends on $\left(A_{1}, A_{2}, Z_{1}, Z_{2}\right)$ as follows

$$
\begin{aligned}
\varepsilon_{i}^{z}\left(A_{1}, A_{2}, Z_{1}, Z_{2}\right)=\operatorname{tr}\left[B_{i} H_{i i}^{I} Z_{i} H_{i i}^{I^{\dagger}} B_{i}^{\dagger}\right] & +\operatorname{tr}\left[B_{i} H_{i j}^{I} Z_{j} H_{i j}^{I^{\dagger}} B_{i}^{\dagger}\right] \\
& -2 \operatorname{Re}\left(\operatorname{tr}\left[A_{i}^{\dagger} H_{i i}^{I^{\dagger}} B_{i}^{\dagger}\right]\right),
\end{aligned}
$$

where $\operatorname{Re}[z]$ denotes the real part of $z \in \mathbb{C}$. Hence the optimization problem in (26) can be reformulated as follows:

$$
\begin{array}{ll}
\min _{\substack{A_{1}, A_{2}, Z_{1}, Z_{2}}} & \alpha_{1} \varepsilon_{1}^{Z}\left(A_{1}, A_{2}, Z_{1}, Z_{2}\right)+\alpha_{2} \varepsilon_{2}^{z}\left(A_{1}, A_{2}, Z_{1}, Z_{2}\right) \\
\text { s.t. } & \mathcal{J}_{1}^{K}\left(Z_{1}, Z_{2}\right) \geq \gamma_{1}, \quad \mathcal{J}_{2}^{K}\left(Z_{1}, Z_{2}\right) \geq \gamma_{2}, \\
& \operatorname{tr}\left[Z_{1}\right] \leq P_{1}, \quad \operatorname{tr}\left[Z_{2}\right] \leq P_{2}, \\
& Z_{1}=A_{1} A_{1}^{\dagger}, \quad Z_{2}=A_{2} A_{2}^{\dagger} .
\end{array}
$$

The constraints in (27d) are not convex, since they represent equality constraints involving a convex function of the variables. Except the constraints in (27d), all constraints are now linear functions of the variables $\left(A_{1}, A_{2}, Z_{1}, Z_{2}\right)$, hence this formulation would constitute a convex problem if the equality constraints in (27d) were not there. We relax these as follows:

$$
Z_{i} \succeq A_{1} A_{1}^{\dagger}, \quad Z_{2} \succeq A_{2} A_{2}^{\dagger} .
$$

By using Schur complement [32, A.5.5], one can equivalently write the expressions in (28) as linear matrix inequalities

$$
S_{1}=\left[\begin{array}{cc}
I & A_{1}^{\dagger} \\
A_{1} & Z_{1}
\end{array}\right] \succeq 0, \quad S_{2}=\left[\begin{array}{cc}
I & A_{2}^{\dagger} \\
A_{2} & Z_{2}
\end{array}\right] \succeq 0 .
$$

Hence the relaxed version of the problem in (27) can be expressed as

$$
\begin{array}{cl}
\min _{\substack{A_{1}, A_{2}, Z_{1} \geq 0, Z_{2} \geq 0}} & \alpha_{1} \varepsilon_{1}^{z}\left(A_{1}, A_{2}, Z_{1}, Z_{2}\right)+\alpha_{2} \varepsilon_{2}^{z}\left(A_{1}, A_{2}, Z_{1}, Z_{2}\right) \\
\text { s.t. } & (27 \mathrm{~b}),(27 \mathrm{c}),(29) .
\end{array}
$$

This is a convex optimization problem, hence it can be solved efficiently by standard numerical optimization tools. We observe that since the optimization in the formulation in (30) is done over a larger set than the formulation in (27), solution of (30) provides a lower bound for the solution of (27). The next theorem shows that a stronger result is true.

Theorem 6.1: Let $n \geq 2$ where $s_{i} \in \mathbb{C}^{n \times 1}, i=1$, 2. Let (30) be solvable. Then the optimum error values for (26) and the relaxed problem in (30) are equal and can be attained. Moreover, an optimal solution for (26) can be constructed from an optimal solution of (30).

The proof is given in Appendix B. This result shows that one can guarantee to find the optimal value for (27) (equivalently (26)) using (30) under solvability of the relaxed problem. Furthermore, a solution to the original problem (a solution satisfying (27d)) can be constructed from an optimal solution for the relaxed problem. Hence although fixed receivers problem is non-convex, Thm. 6.1 guarantees that it can be efficiently solved using a convex problem.

To find a solution for (27) from an optimal solution for (30), following approach is adopted. Let $V^{*}=\left(A_{1}^{*}, A_{2}^{*}, Z_{1}^{*}, Z_{2}^{*}\right)$ denote an optimal solution to (30). We output $A_{1}^{*}, A_{2}^{*}$ as a solution to (27) if the following condition is satisfied,

$$
\mathcal{J}_{i}\left(A_{1}^{*}, A_{2}^{*}\right) \geq \gamma_{i}, \quad i=1,2 .
$$

We note that it is guaranteed that the transmit power conditions are satisfied, and the error values are non-increasing if $Z_{i}^{*}$ is replaced with $A_{i}^{*} A_{i}^{* \dagger}$, due to the conditions $Z_{i} \succeq A_{i} A_{i}^{\dagger}, i=1,2$. Together with the optimality of $V^{*}$ for $(30),\left(A_{1}^{*}, A_{2}^{*}\right)$ is optimal for (27). If (31) is violated, a rank constrained solution for (27) is generated using [27, Algorithm RED], [28, Algorithm 1]. Details can be found in Appendix B.

\section{B. MMSE Estimator at the Receiver}

We now consider the case where MMSE estimators are employed at the receivers. In order to solve the resulting non-convex problem, i.e. Problem $P 1$, we propose a block coordinate-descent method where we take turns in fixing the precoder matrices and the estimators.

For fixed linear precoders $\left(A_{1}, A_{2}\right)$, the problem of finding the MMSE estimators is the classical MMSE estimation problem, and the optimal $B_{i}$ 's are given by (3), [25, Ch. 2],

$$
\begin{aligned}
B_{i} & =K_{s_{i} y_{i}^{I}} K_{y_{i}^{I}}^{-1}, \\
& =A_{i}^{\dagger} H_{i i}^{I^{\dagger}}\left(T_{i}+\sigma_{w, I, i}^{2} I\right)^{-1},
\end{aligned}
$$

where $T_{i}=H_{i i}^{I} A_{i} A_{i}^{\dagger} H_{i i}^{I^{\dagger}}+H_{i j}^{I} A_{j} A_{j}^{\dagger} H_{i j}^{I^{\dagger}}$, and $i, j=1,2, i \neq j$ as before. To find linear precoders for fixed receivers, we solve the problem in (30). To initialize the algorithm, we solve the following problem which maximizes the energy harvested

$$
\begin{array}{ll}
\max _{A_{1}, A_{2}} & \mathcal{J}_{1}\left(A_{1}, A_{2}\right)+\mathcal{J}_{2}\left(A_{1}, A_{2}\right) \\
\text { s.t. } & (6 \mathrm{~b}),(6 \mathrm{c}) .
\end{array}
$$


The resulting method is summarized in Algorithm 1.

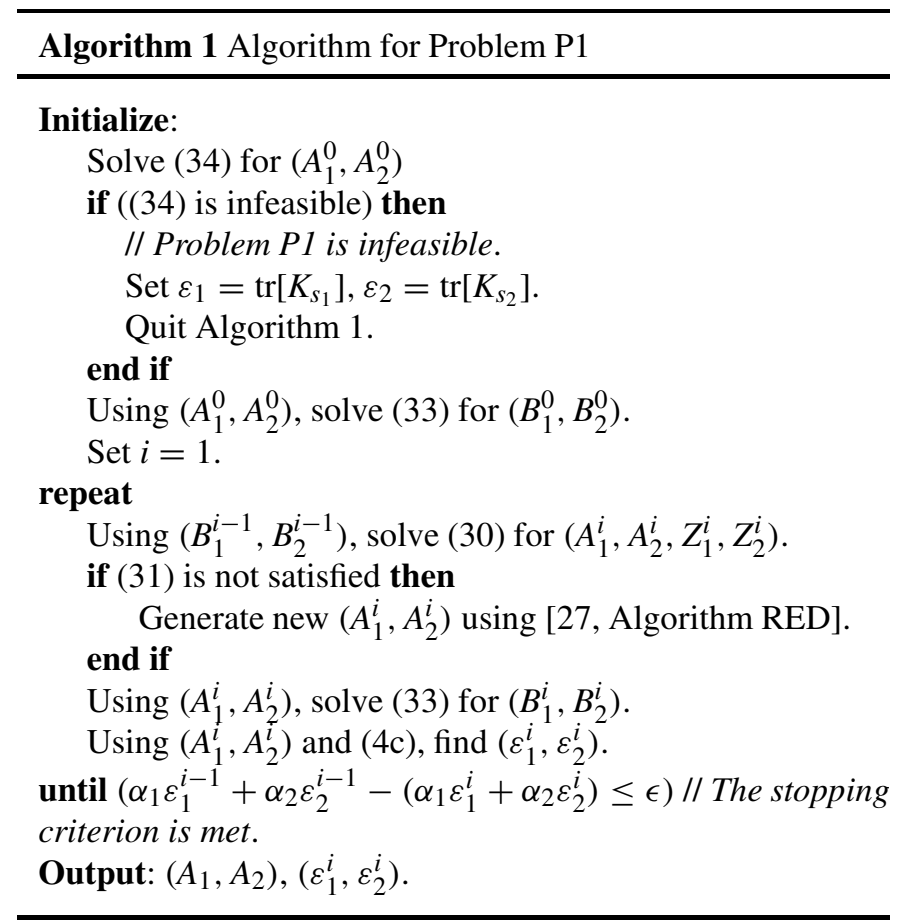

We now discuss the convergence of this method. At each fixed $\left(A_{1}, A_{2}\right)$ step, the estimators are found optimally according to (33). As shown in Theorem 6.1, at each step where the receiver filters $\left(B_{1}, B_{2}\right)$ are fixed, the problem can be optimally solved using a convex problem whenever the original problem is feasible. Hence the objective function is guaranteed to decrease under each iteration. Since the error is bounded from below, Algorithm 1 is guaranteed to converge. We note that due to non-convexity of Problem P1, the optimality of the proposed solutions obtained by Algorithm 1 cannot be guaranteed. Hence they give achievable, but possibly sub-optimal solutions. Nevertheless, for the MISO case, our numerical experiments illustrate that the optimal error values provided by Algorithm 1 coincides with the values provided by the approach discussed in Section V, which is designed specifically for the MISO case and reduces the problem to a line search. Hence with its consistent results in the MISO case, and the general convergence guarantee, Algorithm 1 offers a promising design framework for the joint linear precoder design problem.

We note that each step in the algorithm can be done in polynomial complexity, which includes the solution of the SDP [29], [40], finding a rank constrained solution whose complexity is dominated by the complexity of the solution of a system of linear equations [27, Algorithm RED-step (c)] and finding the estimators through (33) or by solving the corresponding system of linear equations. Required number of iterations is discussed in more detail in Section VIII.

\section{Hybrid TRANSMISSION STRATEgIES}

Here we propose a power splitting scheme at the transmitter, where the transmitted signal is the superposition of two signals one of which is chosen to be a deterministic signal. In
Section VIII, we illustrate that this hybrid scheme allows us to obtain significant improvements over sole Gaussian signalling, see also [9] for similar discussions for rate maximization. These results suggest that, even under the assumption of receivers that can simultaneously decode information and harvest energy, transmission strategies should be restructured in order to go beyond what Gaussian signalling offers.

We consider the following scheme as the transmission strategy

$$
x_{i}=A_{i} s_{i}+g_{i},
$$

where $g_{i} \in \mathbb{C}^{n_{t} \times 1}$ is a deterministic signal that is known at the transmitters and the receivers. The sole purpose of $g_{i}$ is to transfer energy, whereas the purpose of $A_{i} s_{i}$ is to transfer information and also possibly energy. The IRs will be able to perform the MMSE estimation after removing the known interference $g_{i}$ 's. Hence here $g_{i}$ 's do not degrade the performance of the unintended IRs as opposed to the case of using all the power for sending information signal. On the other hand, $g_{i}$ uses some of the power that could have been allocated to the information signal at the transmitter $i$, which may degrade the error performance at $\mathrm{IR}_{i}$.

To find the optimal power allocation trade-off between the energy components $\left(g_{i}\right)$ and information carrying components $\left(A_{i} s_{i}\right)$, along with the optimal waveforms $\left(g_{1}, g_{2}\right)$ and the precoders $\left(A_{1}, A_{2}\right)$, we formulate an optimization problem similar to Problem P1. The power constraint at transmitter $i$ takes the following form

$$
\operatorname{tr}\left[g_{i} g_{i}^{\dagger}\right]+\operatorname{tr}\left[A_{i} A_{i}^{\dagger}\right] \leq P_{i}, \quad i=1,2 .
$$

The energy harvesting constraints can be expressed as follows

$$
\mathcal{J}_{i}\left(A_{1}, A_{2}\right)+\mathcal{J}_{i}\left(g_{1}, g_{2}\right) \geq \gamma_{i}, \quad i=1,2 .
$$

The resulting optimal precoder design problem is the following

$$
\begin{array}{cl}
\left(\mathrm{P} 1_{G D}\right) \min _{g_{1}, g_{2}, A_{1}, A_{2},} & \alpha_{1} \varepsilon_{1}\left(A_{1}, A_{2}\right)+\alpha_{2} \varepsilon_{2}\left(A_{1}, A_{2}\right) \\
\text { s.t. } & (36),(37) .
\end{array}
$$

We note that Problem P1 can be considered as a special case of Problem P $1_{G D}$ with $g_{1}=0, g_{2}=0$. Here the MMSE estimators are again given by (33) since the estimation is done after removing the known interference $g_{i}$. We note that $g_{i}$ 's can be calculated by the IRs using the channel state information of the nodes in the system, which is also used for implementing the MMSE estimation.

We adopt the two step approach in Section VI to solve Problem $P 1_{G D}$. For the fixed estimator step where the receivers use fixed filters $B_{i}, i=1,2$, the following problem is considered

$$
\min _{A_{1}, A_{2}, g_{1}, g_{2}} \alpha_{1} \varepsilon_{1}^{F}+\alpha_{2} \varepsilon_{2}^{F}
$$

subject to (36) and (37). Introducing the variables $G_{i}=g_{i} g_{i}^{\dagger}$, $Z_{i}=A_{i} A_{i}^{\dagger}$, (36) can be expressed as follows

$$
\operatorname{tr}\left[G_{i}\right]+\operatorname{tr}\left[Z_{i}\right] \leq P_{i}, \quad i=1,2 .
$$


The energy harvesting constraints can be written as follows

$$
\mathcal{J}_{i}^{K}\left(Z_{1}, Z_{2}\right)+\mathcal{J}_{i}^{K}\left(G_{1}, G_{2}\right) \geq \gamma_{i}, \quad i=1,2 .
$$

Hence (39) can be expressed as follows

$$
\begin{aligned}
& \min _{\substack{A_{1}, A_{2}, Z_{1}, Z_{2}, g_{1}, g_{2}, G_{1}, G_{2}}} \alpha_{1} \varepsilon_{1}^{F}+\alpha_{2} \varepsilon_{2}^{F} \\
& \text { s.t. } \\
& \quad(40),(41) \\
& G_{i}=g_{i} g_{i}^{\dagger}, \quad Z_{i}=A_{i} A_{i}^{\dagger}, \quad i=1,2 .
\end{aligned}
$$

Using the relaxation in (28), (or equivalently (29)) and lifting the rank constraint on $G_{i}$ 's, we relax this problem as follows

$$
\min _{\substack{A_{1}, A_{2}, Z_{1} \geq 0, Z_{2} \geq 0, G_{1} \succeq 0, G_{2} \succeq 0}} \alpha_{1} \varepsilon_{1}^{z}\left(A_{1}, A_{2}, Z_{1}, Z_{2}\right)+\alpha_{2} \varepsilon_{2}^{z}\left(A_{1}, A_{2}, Z_{1}, Z_{2}\right)
$$

subject to (29), (40), (41). The following relationship holds between the solutions of the original fixed estimator problem (39) and the relaxed problem (43):

Theorem 7.1: Let $n \geq 2$. Let (43) be solvable. Then (39) has the same optimal value with (43). An optimal solution for (39) can be constructed from a solution of (43).

The proof is given in Appendix $\mathrm{C}$. The above result shows that one can effectively solve (43) instead of (39) for the fixed estimator problem. Now the two step procedure in Algorithm 1 can be modified by replacing the step that solves (30) with a step that solves (43) to find designs for the MMSE receiver problem with hybrid signalling in (38). Similar to the sole Gaussian signalling case, the error is non-increasing at each iteration, hence the modified algorithm is guaranteed to converge.

\section{NUMERICAL RESUlTS}

We now illustrate the performance of our designs and the trade-off between the error and the energy harvested through numerical results. The error performance is reported as the weighted sum of normalized MMSEs as follows:

$$
\bar{\varepsilon}=\frac{\alpha_{1} \varepsilon_{1}+\alpha_{2} \varepsilon_{2}}{\varepsilon_{0}},
$$

where $\varepsilon_{0}=\operatorname{tr}\left[K_{s_{1}}\right]=\operatorname{tr}\left[K_{s_{2}}\right]=n$. We choose $\alpha_{1}=\alpha_{2}=1$. When the problem is not feasible, i.e. the EH constraints cannot be satisfied under the given power constraints, the transmission does not occur; hence the error values are set to $\varepsilon_{1}=\varepsilon_{2}=\varepsilon_{0}$. We assume that the energy and information receivers are colocated, $H_{i j}^{I}=H_{i j}^{E}=H_{i j}, i, j=1,2$. We generate the channel matrices independently with i.i.d. complex proper zero-mean Gaussian components with variance $\sigma_{H}^{2}=1$. We report the average results for 100 channel realizations. We set $\epsilon=10^{-5} \varepsilon_{0}$ and $\mathrm{SNR}=10 \mathrm{~dB}$, where $\mathrm{SNR}$ is defined as $\sigma_{H}^{2} / \sigma_{w}^{2}$ with $\sigma_{w, I, i}^{2}=\sigma_{w, E, i}^{2}=\sigma_{w}^{2}, i=1,2$. The EH constraints are set as $\gamma_{1}=\gamma_{2}=\gamma$ (Watts). We assume that the system parameters, including the power constraints and the EH constraints, are scaled to the proper ranges. Discussions on the admissible values can be found in [3], [8]. The convex optimization problems including (30), (34), and (43) are solved using [33]-[35]. Convergence behavior of Algorithm 1 is further discussed at the end of this section.
We label the transmission strategies as follows: $\mathrm{TX}_{G}$ is the proposed design for Problem P1 (Gaussian signalling) found by the approach in Section VI. TX $G D$ is for the hybrid signalling framework in Problem $\mathrm{P} 1_{G D}$ (Gaussian+deterministic signalling) found by the approach in Section VII. We also compare the performance of our joint design strategy with that of individual design where transmitters decide on their transmission strategies independently without any cooperation $\left(\mathrm{TX}_{I N D}\right)$. Here each transmitter assumes there is no interference and aims to minimize the MMSE at its designated IR under the EH constraint at its designated ER.

We also compare the performance of our designs with that of TDMA and TDMS of [7]. In both schemes, transmission interval is divided into two time slots. In TDMA, for $0 \leq t_{a} \leq 1$ fraction of the time, the system operates in $(I, E)$ mode: Receiver 1 operates in the information decoding (ID) mode whereas Receiver 2 operates in the $\mathrm{EH}$ mode. In the remaining $1-t_{a}$ fraction of the time, the operating mode is $(E, I)$ where the roles of the receivers are swapped. In TDMS, for $0 \leq t_{s} \leq 1$ fraction of time, both receivers operate in the $\mathrm{EH}$ mode $(E, E)$. In the remaining $1-t_{s}$ fraction of time, both receivers operate in the ID mode $(I, I)$. As previously mentioned, these comparisons are motivated by the fact that schemes that depend on such mode separations have been considered as practical benchmarks in the context of rate maximization [7]-[10]. Here we adopt these schemes to the MMSE minimization problem. For TDMA, transmitters adopt deterministic signalling when their intended receiver operates in the EH mode. For optimization over the parameter $t_{a}$, we adopt a line search over the set $S_{t_{a}}=\{0.05 k: k=0, \ldots, 20\}$. We note that Algorithm 1 can be used to find the transmission strategy for the transmitter serving to both IR and ER receiver, for instance transmitter 1 in $(I, E)$ mode. Nevertheless, for the sake of reduced computation time, we have preferred to solve the relaxed problem in (16). Hence TDMA curves we present here are lower bounds on TDMA performance on $S_{t_{a}}$. For TDMS, optimum time-sharing parameter $t_{s}$ is found using a convex optimization approach similar to [7, Sec. IV-A].

We first study the MMSE performance difference between the rate maximization and the mean-square error minimization problems. Our aim is to illustrate that these two metrics, (rate and the MMSE) are although closely related, lead to different MMSE performance trade-offs. Without the EH constraints, it is known that the MMSE minimization and rate maximization problems have different optimal solutions, see for instance [28, Table 3.1]. The difference in the form of optimal solutions under EH constraints can be seen by comparing Thm. 4.1 here and [3, Thm. 3.1]. Here we present a numerical qualification of the resulting performance difference. We focus on the P2P case with $n_{t} \leq n$ so that the results can be fully attributed to the difference between the optimal solutions without any reference to possibly sub-optimal approaches we will have to refer to in the case of interference channel and the rank constrained scenarios. Hence, for the MMSE minimization, we consider the formulation in (15). For the rate maximization problem, we consider the following problem

$$
\max _{K_{1} \succeq 0} \log \left(\left|I+\frac{1}{\sigma_{w, I, 1}^{2}} H_{11}^{I} K_{1} H_{11}^{I^{\dagger}}\right|\right)
$$




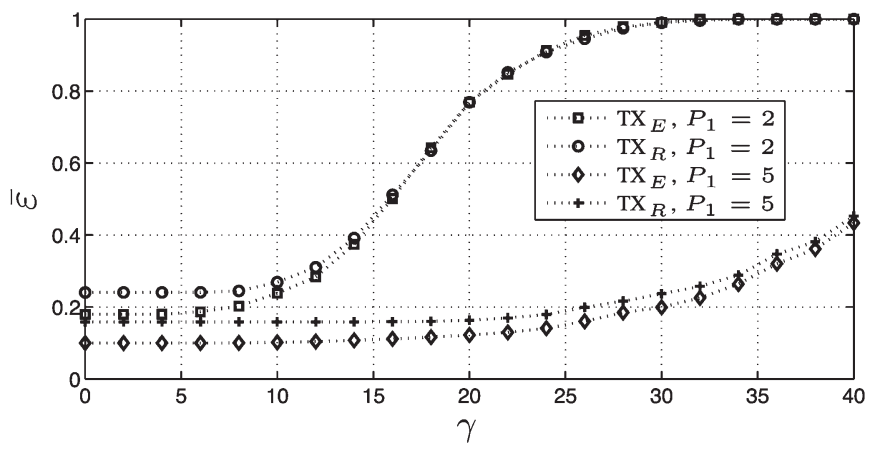

Fig. 2. MMSE versus energy harvesting requirements, comparison of the MMSE performance of the rate maximization and the MMSE minimization schemes.

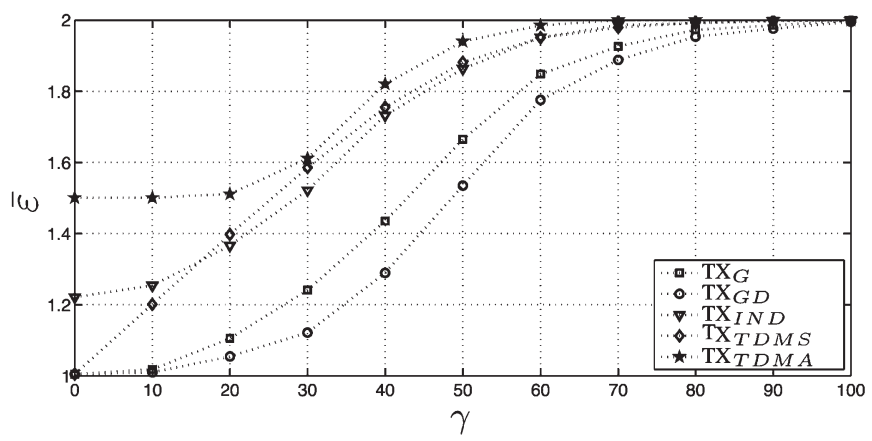

Fig. 3. MMSE versus energy harvesting requirements, $n=2, n_{t}=4, n_{r}=$ $n_{e}=1,\left(P_{1}, P_{2}\right)=(10,10)$.

under the conditions $\operatorname{tr}\left[H_{11}^{E} K_{1} H_{11}^{E^{\dagger}}\right] \geq \gamma_{1}$ and $\operatorname{tr}\left[K_{1}\right] \leq P_{1}$. This problem is studied in [3]. Both of these problems are convex and are solved using [33]-[35]. The MMSE performance of the transmission schemes using the resulting optimum transmit covariance matrices $K_{1}$ are presented in Fig. 2 averaged over different channel realizations. Here $T X_{E}$ and $T X_{R}$ correspond to the performance of the solution of (16) and (45), respectively. We have $n_{t}=n_{i}=n_{e}=n=4$. We observe that the relative difference is substantial under small and moderate EH constraints. To quantify this, let us define the relative performance difference ratio $r=100\left(\varepsilon_{T X_{R}}-\varepsilon_{T X_{E}}\right) / \varepsilon_{T X_{E}}$, where $\varepsilon_{T X_{S}}$ is the error associated with transmission strategy $S \in\{R, E\}$. For instance, for the $\mathrm{EH}$ constraint $\gamma=8$, the relative performance difference is $r \approx 20 \%$ and $r \approx 58 \%$ for $P_{1}=2$ and $P_{1}=5$, respectively. With more demanding EH constraints there is little room for error minimization or rate maximization, hence the performance gap gets smaller.

We now consider the MISO interference scenario with $n=2$, $n_{t}=4, n_{r}=n_{e}=1$. Figs. 3 and 4 show the error versus the EH constraint curves for $\left(P_{1}, P_{2}\right)=(10,10)$ and $\left(P_{1}, P_{2}\right)=$ $(2,18)$, respectively. We observe that as expected, for all power budget pairs, error increases as the EH requirements become more demanding. TDMA and TDMS approaches exhibit significantly weak performance compared to $\mathrm{TX}_{G}$ and $\mathrm{TX}_{G D}$, especially for low to moderate EH values. This effect is particularly prominent for the unbalanced power budget case (Fig. 4). These observations confirm the need for the design of novel transmission strategies. The plots also illustrate that the joint design schemes $T X_{G}$ and $T X_{G D}$ perform substantially better than the

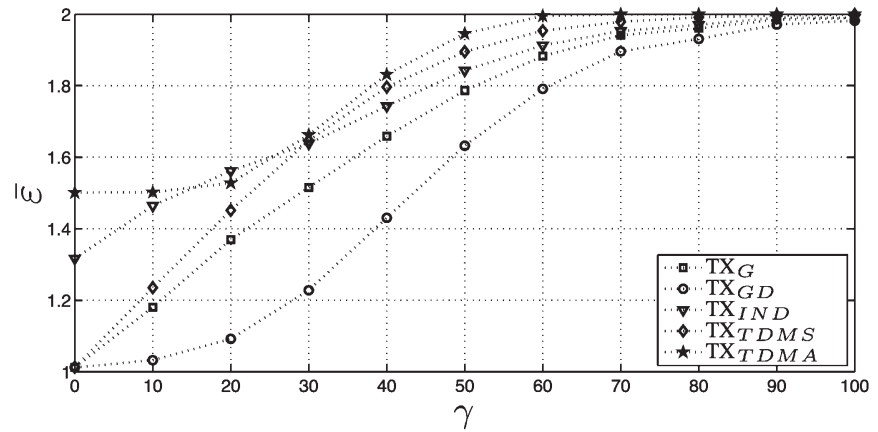

Fig. 4. MMSE versus energy harvesting requirements, $n=2, n_{t}=4, n_{r}=$ $n_{e}=1,\left(P_{1}, P_{2}\right)=(2,18)$.

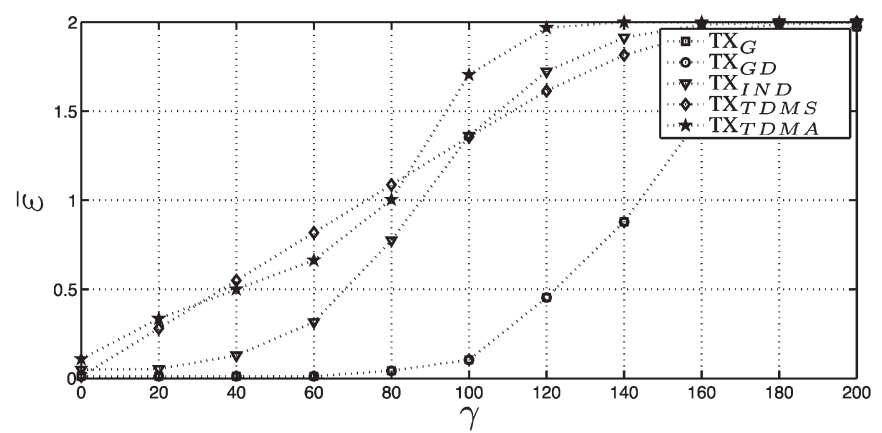

Fig. 5. MMSE versus energy harvesting requirements, $n=2, n_{t}=4, n_{r}=$ $n_{e}=4,\left(P_{1}, P_{2}\right)=(10,10)$.

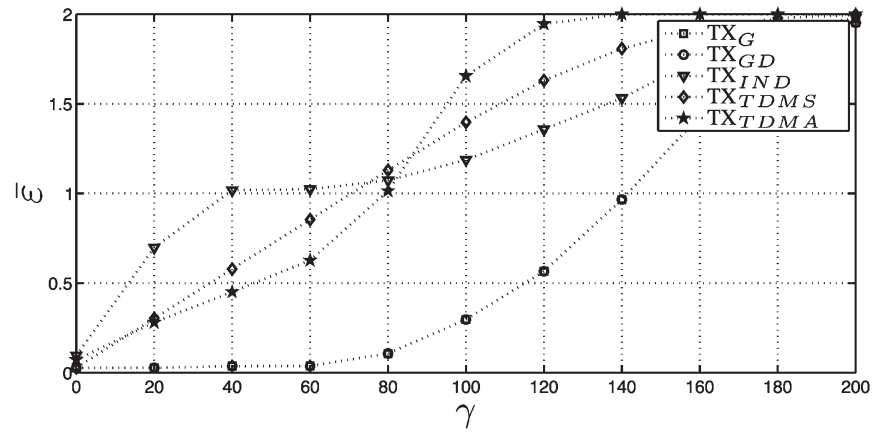

Fig. 6. MMSE versus energy harvesting requirements, $n=2, n_{t}=4, n_{r}=$ $n_{e}=4,\left(P_{1}, P_{2}\right)=(2,18)$.

independent design scheme $T X_{I N D}$. Comparing the performance of $T X_{G}$ and $T X_{G D}$ for fixed transmission power budget, we observe that significant gains can be obtained by adopting the hybrid scheme. Comparing the results in Figs. 3 and 4 we observe that it is possible to obtain lower error values when the power budget pairs are more balanced, i.e., both of the users have equal or close transmission power budgets. This is consistent with the fact that channel conditions are symmetric and the EH demands are equal.

We now consider the MIMO channel case with $n=2, n_{t}=$ $4, n_{r}=n_{e}=4$. Figs. 5 and 6 show the error versus the EH constraint curves for $\left(P_{1}, P_{2}\right)=(10,10)$ and $\left(P_{1}, P_{2}\right)=(2,18)$, respectively. Compared to the previous MISO scenarios, for all transmission strategies, it is observed that it is possible to obtain lower values of error for a given EH constraint. This performance improvement is consistent with the higher number of degrees of freedom offered by the multiple antennas at the 


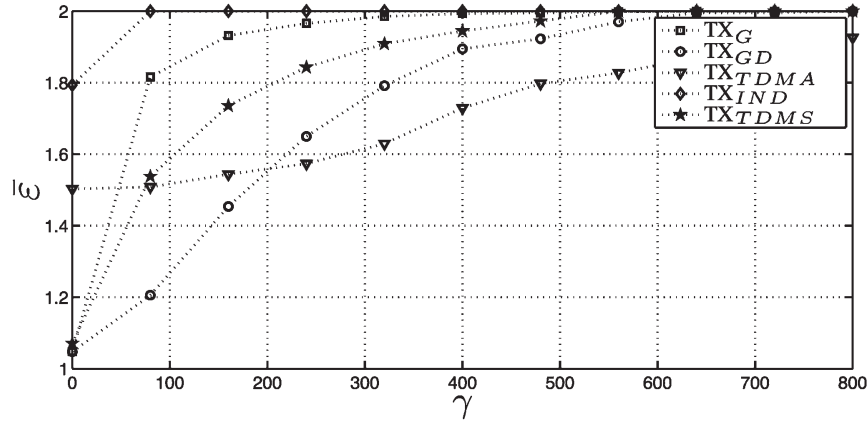

Fig. 7. MMSE versus energy harvesting requirements. High interference scenario.

receivers. Due to these extra degrees of freedom, the transmitters can better shape their transmissions so that the interference to the unintended information receivers can be kept low. This also contributes to the decreasing performance difference between $\mathrm{TX}_{G}$ and $\mathrm{TX}_{G D}$ as the number of antennas increases; $\mathrm{TX}_{G D}$ does not offer any significant gains over $\mathrm{TX}_{G}$ for $n_{r}=4$. The extra antennas at the ERs also allow the receivers to harvest the energy in the signals that can arrive at the receiver through these extra paths, so higher values of energy can be harvested.

We study the effect of the level of cross-interference on the trade-offs in Fig. 7. To quantify the level of cross-interference, a scaling parameter $\mu$ is used where the cross-channel matrices are scaled as $\mu H_{12}$ and $\mu H_{21}$. We set $n=2, n_{t}=2$, $n_{r}=n_{e}=1,\left(P_{1}, P_{2}\right)=(10,10)$ and $\mu=4$. We observe that TDMA can outperform both $\mathrm{TX}_{G}$ and $\mathrm{TX}_{G D}$ and TDMS can outperform $\mathrm{TX}_{G}$. (Since there are no rank constraints, here TDMA performance is the true performance on the set $S_{t_{a}}$ rather than a lower bound.) We note that the superior performance of TDMA is not a characteristics specific to the energy harvesting problem. Under heavy interference, better MMSE values can be obtained by the TDMA approach compared to the optimized signalling, even when there are no EH constraints. This can be seen, for instance, by considering the scalar channel case and letting the cross-link powers go to infinity. On the other hand, TDMS cannot outperform $\mathrm{TX}_{G D}$. This is an analytical property of TDMS and $\mathrm{TX}_{G D}$ : Let $0 \leq t_{s} \leq 1$ be the fraction of time spent in energy transfer in TDMS. Then any error value that is achievable by TDMS using $t_{s}$ will be also achievable by $\mathrm{TX}_{G D}$ by using $t_{s}$ fraction of the power available for energy transfer with deterministic signalling.

The convergence behavior of Algorithm 1 is illustrated in Fig. 8. The MMSE versus iteration index curves are presented for $P_{1}=P_{2}=10$ for $n=2, n_{t}=4, n_{r}=n_{e}$ for $\mathrm{TX}_{G}$ and $\mathrm{TX}_{G D}$ for $\gamma=50$ and $\gamma=100$, for $n_{r}=1$ and $n_{r}=4$, respectively. For each case, curves for three different channel realizations are plotted. In Table I, we also give the average number of iterations for the scenarios presented in Figs. 3-6, where the stopping tolerance is $\epsilon=10^{-5} \varepsilon_{0}$. The convergence is observed to be pretty rapid, especially in the $n_{r}=1$ scenario. In general, the EH constraints also affect the number of iterations. For $n_{r}=1, P_{1}=P_{2}=10, \mathrm{TX}_{G}$ shows no significant dependence on $\gamma$, whereas the average number of iterations increases as $\gamma$ increases for $\mathrm{TX}_{G D}$. For instance, for $\mathrm{TX}_{G D}$ approximately 8 and 17 iterations are needed on average for $\gamma=20$ and

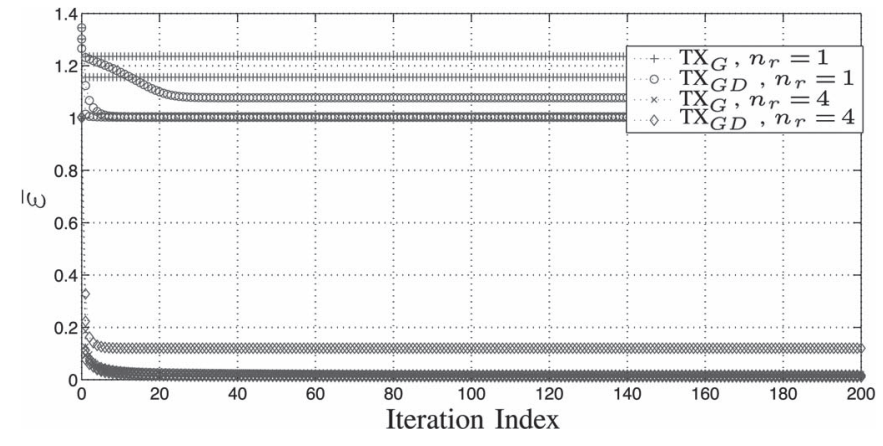

Fig. 8. Convergence behavior of Algorithm 1.

TABLE I

AVErage Number of ITERATIONS FOR ALgORITHM 1

\begin{tabular}{|l|c|c|}
\hline & $\mathrm{TX}_{G}$ & $\mathrm{TX}_{G D}$ \\
\hline$n_{r}=n_{e}=1,\left(P_{1}, P_{2}\right)=(10,10)$ & 5.14 & 15.45 \\
\hline$n_{r}=n_{e}=1,\left(P_{1}, P_{2}\right)=(2,18)$ & 5.89 & 15.44 \\
\hline$n_{r}=n_{e}=4,\left(P_{1}, P_{2}\right)=(10,10)$ & 74.92 & 75.58 \\
\hline$n_{r}=n_{e}=4,\left(P_{1}, P_{2}\right)=(2,18)$ & 105.59 & 109.37 \\
\hline
\end{tabular}

$\gamma=60$. For $n_{r}=4, P_{1}=P_{2}=10$, the general behaviour of both schemes are the same; the average number of iterations decreases as $\gamma$ increases. For instance, approximately 90 and 60 iterations are needed on average for $\gamma=40$ and $\gamma=160$.

\section{CONCLUSION}

We have considered the problem of linear precoder design with the aim of minimizing the sum MMSE in MIMO interference channels with energy harvesting constraints. In the case where there is only one user, i.e. for the P2P channel, the problem reduces to a convex problem under a constraint on the transmit antennas. For this case, we have provided a characterization of the optimum solutions. For the general interference scenario, the problem leads to a non-convex formulation for the solution of which we have proposed an efficient numerical approach. We have also investigated a hybrid signalling scheme, where the transmitters send a superposition of two signals: a deterministic signal optimized for energy transfer and an information carrying signal optimized for information and energy transfer. It is illustrated that hybrid signalling offers significant gains over sole Gaussian signalling when the number of antennas at the receivers are relatively small.

\section{APPENDIX A}

\section{Proof OF LEMMA 5.1}

Let us consider the inner optimization problem in (25) obtained by fixing $t$, which is a SDP problem with six constraints (other than positive semi-definiteness constraints). By [28, Thm. 3.2], there exists an optimal solution which satisfies $\operatorname{rank}\left(\bar{K}_{i}^{*}\right)^{2}+\operatorname{rank}\left(\bar{K}_{2}^{*}\right)^{2} \leq 6$. Hence $\operatorname{rank}\left(\bar{K}_{i}^{*}\right)=\operatorname{rank}\left(K_{i}^{*}\right) \leq 2$. Hence under the condition $n \geq 2$, an $A_{i}^{*} \in \mathbb{C}^{n_{t} \times n}$ satisfying $K_{i}=A_{i}^{*} A_{i}^{* \dagger}$ can be always formed. (We note that the condition $n \geq 2$ is merely a sufficient condition that guarantees existence of an admissible $A_{i}$.) Such a solution can be constructed 
using [27, Algorithm RED], [28, Algorithm 1]. We finish the proof by noting that since this above argument is true for an arbitrary $t$, it is also true for an optimal $t^{*}$. We note that if the optimal solutions have rank smaller than $n$, during linear precoding only $\operatorname{rank}\left(K_{i}\right)$ of the data streams will be sent. This is similar to water-filling type solutions for MMSE minimization under sole transmission power constraints, see for instance [16] for further discussions.

\section{APENDIX B}

\section{PROOF OF THM. 6.1}

The proof relies on considering the individual optimization problems in (26) obtained by fixing $A_{1}$ or $A_{2}$, and the results on semi-definite programming relaxations of QCQP problems with one matrix variable. Let $\left(A_{1}^{*}, A_{2}^{*}, Z_{1}^{*}, Z_{2}^{*}\right)$ be an optimal solution of (30). Let us consider (26) with fixed $A_{2}^{*}, Z_{2}^{*}$

$$
\begin{array}{ll}
\min _{A_{1}} & f_{1}\left(A_{1}\right) \\
\text { s.t. } & \operatorname{tr}\left[A_{1}^{\dagger} H_{i 1}^{E^{\dagger}} H_{i 1}^{E} A_{1}\right] \geq \bar{\gamma}_{i}, \quad i=1,2 \\
& \operatorname{tr}\left[A_{1} A_{1}^{\dagger}\right] \leq P_{1},
\end{array}
$$

where

$$
\begin{aligned}
f_{1}\left(A_{1}\right) & =\operatorname{tr}\left[A_{1}^{\dagger} C_{1} A_{1}\right]-2 \alpha_{1} \operatorname{Re}\left[\operatorname{tr}\left[A_{1}^{\dagger} H_{11}^{I^{\dagger}} B_{1}^{\dagger}\right]\right], \\
C_{1} & =\alpha_{1} H_{11}^{I^{\dagger}} B_{1}^{\dagger} B_{1} H_{11}^{I}+\alpha_{2} H_{21}^{I^{\dagger}} B_{2}^{\dagger} B_{2} H_{21}^{I}
\end{aligned}
$$

and $\bar{\gamma}_{i}=\gamma_{i}-\operatorname{tr}\left[H_{i 2}^{E^{\dagger}} H_{i 2}^{E} Z_{2}^{*}\right], i=1,2$. Hence for fixed $A_{2}$, (26) is a QCQP problem with a matrix variable and three constraints.

We observe that (30) for fixed $A_{2}^{*}, Z_{2}^{*}$ can be alternatively written in terms of the positive semi-definite variable $S_{1}$ instead of $A_{1}, Z_{1}$. Hence (30) for fixed $A_{2}, Z_{2}$ is in fact the SDP relaxation of (46). (One may refer to [27, 2.7] for the general form of the SDP relaxation of a QCQP problem with matrix variables.) By [27, Thm 2.2], (46) and its SDP relaxation have the same optimal value if the relaxation is solvable and the number of constraints is equal to or smaller than $2 n$. Here the SDP relaxation of (46) is guaranteed to be solvable, since the bi-variate relaxation (30) is assumed to be solvable, and $A_{2}^{*}, Z_{2}^{*}$ is an optimal solution. We observe that the dual of (30) (for fixed $A_{2}^{*}$ ) is strictly feasible since the regularity condition in $[27,2.10]$ holds. (This is due to the fact that the matrix associated with the power constraints, identity, is positive definite.) Hence together with the feasibility of (46), this implies solvability of SDP relaxation [27, Cor. 2.1].

One can utilize the same arguments for the optimization over $A_{2}$ for fixed $A_{1}^{*}$ and its relaxation. We also note that the optimum value for (26) can be found by first optimizing over one variable, and treating the other one fixed, and then optimizing over the second variable. First part of Thm. 6.1 follows from these observations and the above arguments. For the second part, we observe the following: An optimal solution for (26) can be constructed from a solution of (30) using [27, Algorithm RED] (or similarly [28, Algorithm 1]) on $S_{1}$ and $S_{2}$ and by considering the sub-problems for fixed $\left(A_{2}, Z_{2}\right)$ and $\left(A_{1}, Z_{1}\right)$. Due to
[27, Lemma 2.1], desired solution for $A_{i}$ is given by the lower left $n_{t} \times n$ matrix of the rank-constrained $S_{i}$. We note that by construction these algorithms guarantee these sub-matrices satisfy the constraints of the original problem and do not degrade the objective function.

\section{APPENDIX C \\ PROOF OF THM. 7.1}

The proof adopts the same arguments in the proof of Thm. 6.1 in Appendix B. Here we highlight the main differences. Let (30) be solvable, and $V^{*}=\left\{A_{1}^{*}, A_{2}^{*}, Z_{1}^{*}, Z_{2}^{*}, G_{1}^{*}, G_{2}^{*}\right\}$ be an optimal solution. We consider the following feasibility problem over $G_{1}$ when the other variables are kept fixed

$$
\min _{G_{1} \succeq 0} 0
$$

subject to $\operatorname{tr}\left[G_{1}^{\dagger}\right] \leq \bar{P}_{1}$ and $\mathcal{J}_{i}^{K}\left(G_{1}, 0\right) \geq \overline{\gamma_{i}}, i=1,2$. Here $\overline{\gamma_{1}}$, $\overline{\gamma_{2}}$, and $\bar{P}_{1}$ are the modified values of the constraints found by using the optimum values of the variables other than $G_{1}^{*}$, i.e., $V^{*} \backslash\left\{G_{1}^{*}\right\}$. This is a homogeneous QCQP problem with three constraints. We note that (47) is solvable, since (43) is solvable. Hence by [28, Thm 3.2], [27, Thm 2.1], there exists a solution for which $\operatorname{rank}\left(G_{1}\right) \leq 1$. This solution can be constructed by [27, Algorithm RED], [28, Algorithm 1]. By considering the sub-problems obtained by fixing the other variables and utilizing Theorem 6.1, the result follows.

\section{REFERENCES}

[1] L. Varshney, "Transporting information and energy simultaneously," in Proc. IEEE ISIT, Jul. 2008, pp. 1612-1616.

[2] P. Grover and A. Sahai, "Shannon meets Tesla: Wireless information and power transfer," in Proc. IEEE ISIT, Jun. 2010, pp. 2363-2367.

[3] R. Zhang and C. K. Ho, "MIMO broadcasting for simultaneous wireless information and power transfer," IEEE Trans. Wireless Commun., vol. 12, no. 5, pp. 1989-2001, May 2013.

[4] J. Xu, L. Liu, and R. Zhang, "Multiuser MISO beamforming for simultaneous wireless information and power transfer," IEEE Trans. Signal Process., vol. 62, no. 18, pp. 4798-4810, Sep. 2014.

[5] A. Nasir, X. Zhou, S. Durrani, and R. Kennedy, "Relaying protocols for wireless energy harvesting and information processing," IEEE Trans. Wireless Commun., vol. 12, no. 7, pp. 3622-3636, Jul. 2013.

[6] Y. Luo, J. Zhang, and K. Letaief, "Optimal scheduling and power allocation for two-hop energy harvesting communication systems," IEEE Trans. Wireless Commun., vol. 12, no. 7, pp. 4729-4741, Sep. 2013.

[7] C. Shen, W.-C. Li, and T.-H. Chang, "Wireless information and energy transfer in multi-antenna interference channel," IEEE Trans. Signal Process., vol. 62, no. 23, pp. 6249-6264, Dec. 2014.

[8] J. Park and B. Clerckx, "Joint wireless information and energy transfer in a two-user MIMO interference channel," IEEE Trans. Wireless Commun., vol. 12, no. 8, pp. 4210-4221, Aug. 2013.

[9] S. Lee, L. Liu, and R. Zhang, "Collaborative wireless energy and information transfer in interference channel," IEEE Trans. Wireless Commun. vol. 14, no. 1, pp. 545-557, Jan. 2015.

[10] J. Park and B. Clerckx, "Joint wireless information and energy transfer in a $K$-user MIMO interference channel," IEEE Trans. Wireless Commun., vol. 13, no. 10, pp. 5781-5796, Oct. 2014.

[11] K. Huang and E. Larsson, "Simultaneous information and power transfer for broadband wireless systems," IEEE Trans. Signal Process., vol. 61, no. 3, pp. 5972-5986, Dec. 2013.

[12] A. M. Fouladgar, O. Simeone, and E. Erkip, "Constrained codes for joint energy and information transfer," IEEE Trans. Commun., vol. 62, no. 6, pp. 2121-2131, Jun. 2014.

[13] H. Sampath, P. Stoica, and A. Paulraj, "Generalized linear precoder and decoder design for MIMO channels using the weighted MMSE criterion," IEEE Trans. Commun., vol. 49, no. 12, pp. 2198-2206, Dec. 2001. 
[14] M. Rupf, F. Tarkoy, and J. Massey, "User-separating demodulation for code-division multiple-access systems," IEEE J. Sel. Areas Commun., vol. 12, no. 5, pp. 786-795, Jun. 1994.

[15] R. Wang, M. Tao, and Y. Huang, "Linear precoding designs for amplifyand-forward multiuser two-way relay systems," IEEE Trans. Wireless Commun., vol. 11, no. 12, pp. 4457-4469, Dec. 2012.

[16] D. P. Palomar and Y. Jiang, "MIMO transceiver design via majorization theory," Found. Trends Commun. Inf. Theory, vol. 3, no. 4/5, pp. 331-551, Nov. 2007.

[17] H. Shen, J. Wang, W. Xu, Y. Rong, and C. Zhao, "A worst-case robust MMSE transceiver design for nonregenerative MIMO relaying," IEEE Trans. Wireless Commun., vol. 13, no. 2, pp. 695-709, Feb. 2014.

[18] A. Nayyar, T. Başar, D. Teneketzis, and V. Veeravalli, "Optimal strategies for communication and remote estimation with an energy harvesting sensor," IEEE Trans. Autom. Control, vol. 58, no. 9, pp. 2246-2260, Sep. 2013.

[19] N. Roseveare and B. Natarajan, "An alternative perspective on utility maximization in energy-harvesting wireless sensor networks," IEEE Trans. Veh. Technol., vol. 63, no. 1, pp. 344-356, Jan. 2014.

[20] M. Nourian, A. Leong, and S. Dey, "Optimal energy allocation for Kalman filtering over packet dropping links with imperfect acknowledgments and energy harvesting constraints," IEEE Trans. Autom. Control, vol. 59, no. 8, pp. 2128-2143, Aug. 2014

[21] M. R. A. Khandaker and K. Wong, "SWIPT in MISO multicasting systems," IEEE Wireless Commun. Lett., vol. 3, no. 3, pp. 277-280, Jun. 2014.

[22] S. Timotheou, I. Krikidis, and B. Ottersten, "MISO interference channel with QoS and RF energy harvesting constraints," in Proc. IEEE ICC, Jun. 2013, pp. 4191-4196.

[23] Q. Shi, C. Peng, W. Xu, and Y. Wang, "Joint transceiver design for MISO SWIPT interference channel," in Proc. IEEE ICASSP, May 2014, pp. 4753-4757.

[24] Q. Shi, L. Liu, W. Xu, and R. Zhang, "Joint transmit beamforming and receive power splitting for MISO SWIPT systems," IEEE Trans. Wireless Commun., vol. 13, no. 6, pp. 3269-3280, Jun. 2014.

[25] B. D. O. Anderson and J. B. Moore, Optimal Filtering. Upper Saddle River, NJ, USA: Prentice-Hall, 1979.

[26] W. Zhong and J. Wang, "Energy efficient spectrum sharing strategy selection for cognitive MIMO interference channels," IEEE Trans. Signal Process., vol. 61, no. 14, pp. 3705-3717, Jul. 2013.

[27] A. Beck, "Convexity properties associated with nonconvex quadratic matrix functions and applications to quadratic programming," J. Optim. Theory Appl., vol. 142, no. 1, pp. 1-29, Jul. 2009.

[28] Y. Huang and D. Palomar, "Rank-constrained separable semidefinite programming with applications to optimal beamforming," IEEE Trans. Signal Process., vol. 58, no. 2, pp. 664-678, Feb. 2010.

[29] Z.-Q. Luo, W.-K. Ma, A. So, Y. Ye, and S. Zhang, "Semidefinite relaxation of quadratic optimization problems," IEEE Signal Process. Mag., vol. 27, no. 3, pp. 20-34, May 2010.

[30] H. V. Henderson and S. R. Searle, "On deriving the inverse of a sum of matrices," SIAM Review, vol. 23, no. 1, pp. 53-60, Jan. 1981.

[31] A. Kashyap, T. Başar, and R. Srikant, "Minimum distortion transmission of gaussian sources over fading channels," in Proc. IEEE Conf. Decision Control, Dec. 2003, vol. 1, pp. 80-85.

[32] S. Boyd and L. Vandenberghe, Convex Optimization. Cambridge, U.K.: Cambridge Univ. Press, 2004.

[33] J. F. Sturm, "Using SeDuMi 1. 02, a matlab toolbox for optimization over symmetric cones," Optim. Methods Softw., vol. 11, no. 1-4, pp. 625-653, Jan. 1999.

[34] R. H. Tütüncü, K. C. Toh, and M. J. Todd, "Solving semidefinite-quadraticlinear programs using SDPT3," Math. Programm., vol. 95, no. 2, pp. 189 217, Feb. 2003

[35] CVX: Matlab Software for Disciplined Convex Programming 2.0, CVX Research Inc., Austin, TX, USA, 2012. [Online]. Available: http://cvxr. $\operatorname{com} / \mathrm{cvx}$
[36] R. Zhang, Y.-C. Liang, and S. Cui, "Dynamic resource allocation in cognitive radio networks," IEEE Signal Process. Mag., vol. 27, no. 3 , pp. 102-114, May 2010.

[37] Q. Shi, M. Razaviyayn, Z.-Q. Luo, and C. He, "An iteratively weighted MMSE approach to distributed sum-utility maximization for a MIMO interfering broadcast channel," IEEE Trans. Signal Process., vol. 59, no. 9, pp. 4331-4340, Sep. 2011

[38] A. Charnes and W. W. Cooper, "Programming with linear fractional functionals," Naval Res. Logist. Quart., vol. 9, no. 3/4, pp. 181-186, Dec. 1962.

[39] Y. Huang, A. D. Maio, and S. Zhang, "Semidefinite programming, matrix decomposition, and radar code design," in Convex Optimization in Signal Processing and Communications, Y. Eldar and D. P. Palomar, Eds., Cambridge, U.K.: Cambridge Univ. Press, 2009.

[40] C. Helmberg, F. Rendl, R. J. Vanderbei, and H. Wolkowicz, "An interiorpoint method for semidefinite programming," SIAM J. Optim., vol. 6, no. 2, pp. 342-361, May 1996.

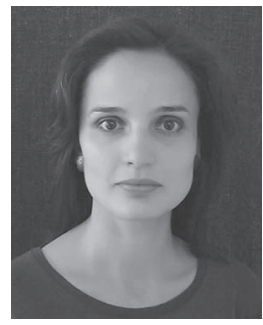

Ayça Özçelikkale (M'10) received the B.Sc. degree in electrical engineering and the double major B.A. degree in philosophy from Middle East Technical University, Ankara, Turkey, and the M.S. and Ph.D. degrees in electrical engineering from Bilkent University, Ankara, Turkey. She spent part of her doctoral studies at the Department of Mathematics and Statistics, Queens University, Kingston, ON, Canada. She is now a Post-doctoral Researcher at the Chalmers University, Gothenburg, Sweden. Her research interests are in the areas of statistical signal processing, communications and optimization.

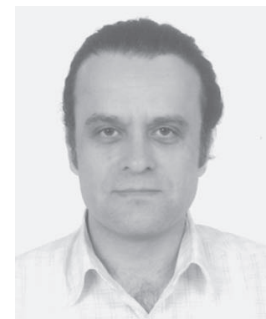

Tolga M. Duman (S'95-M'98-SM'03-F'11) received the B.S. degree from Bilkent University in Turkey, in 1993, and the M.S. and Ph.D. degrees from Northeastern University, Boston, MA, USA, in 1995 and 1998, respectively, all in electrical engineering. He is a Professor in the Electrical and Electronics Engineering Department, Bilkent University, Turkey, and an Adjunct Professor with the School of ECEE, Arizona State University. Prior to joining Bilkent University in September 2012, he was with the Electrical Engineering Department, Arizona State University first as an Assistant Professor (1998-2004), as an Associate Professor (2004-2008), and as a Professor (2008-2015). Dr. Duman's current research interests are in systems, with particular focus on communication and signal processing, including wireless and mobile communications, coding/ modulation, coding for wireless communications, data storage systems and underwater acoustic communications.

Dr. Duman is a recipient of the National Science Foundation CAREER Award and IEEE Third Millennium medal. He served as an editor for IEEE TRANSACTIONS ON WiRELESS COMMUNICATIONS (2003-2008), IEEE TRANSACTIONS ON COMMUNICATIONS (2007-2012) and IEEE ONLINE JOURNAL OF SURVEYS AND TUTORIALS (2002-2007). He is currently the coding and communication theory area editor for IEEE TRANSACTIONS ON Communications (2011-present) and an editor for Elsevier Physical Communications Journal (2010-present). 